\title{
A baixa adesão do protocolo de sepse na terapia intensiva
}

\section{The low adherence of the sepsis protocol in intensive care}

\author{
Rafael Hesley Araujo Costa ${ }^{1}$. Pedro Henrique Sá Costa ${ }^{2}$. André Luis Coutinho de Araújo Macedo ${ }^{3}$. Rafaela \\ Menezes Souza Pessoa ${ }^{4}$. Ianna Lacerda Sampaio Braga ${ }^{5}$. \\ 1 Médico, Residente de Reumatologia, Hospital Geral de Fortaleza (HGF), Fortaleza, Ceará, Brasil. 2 Farmacêutico, Doutorando \\ em Farmacologia, Universidade Federal do Ceará (UFC), Fortaleza, Ceará, Brasil. 3 Médico, Residência em Clínica Médica, \\ Coordenador da Unidade de Terapia Intensiva do Hospital Geral Cesar Cals de Oliveira, Fortaleza, Ceará, Brasil. 4 Médica, \\ Residência em Clínica Médica, Hospital Geral Cesar Cals de Oliveira, Fortaleza, Ceará, Brasil. 5 Médica, Mestre em Biologia \\ Estrutural e Funcional, Professora do curso de Medicina pela Universidade de Fortaleza (UNIFOR), Fortaleza, Ceará, Brasil.
}

\section{RESUMO}

Objetivo: avaliar os prontuários dos pacientes com sepse na unidade de terapia intensiva (UTI) de um hospital terciário, mensurando o grau de adesão ao protocolo de sepse do Ministério da Saúde. Método: este trabalho constitui um estudo transversal, do tipo descritivo, em que foi determinada a frequência de adesão às medidas de cuidados intensivos nas primeiras 6 horas após diagnóstico de sepse. Resultados: observou-se predominância de pacientes do sexo masculino. A média de idade foi de $47 \pm 21$ anos. Do total de pacientes, 14 (28\%) foram acometidos por sepse, 21 (42\%) por sepse grave e 15 (30\%) por choque séptico. Nestes pacientes, foram verificados realização de hemocultura em 28 (56\%), aferição da pressão venosa central (PVC) em 47 (94\%), determinação do índice de saturação venosa de oxigênio $\left(\mathrm{SVO}_{2}\right)$ em $5(10 \%)$ e dosagem de lactato plasmático em 42 (84\%). Ao final, observou-se que em nenhum dos pacientes todas as condutas do protocolo foram realizadas, e em 4 (8\%) destes foi implementado $80 \%$ do protocolo. Conclusão: diante da não adesão integral às medidas propostas no presente protocolo, faz-se necessário um maior investimento na formação de equipes aptas a realizar suas estratégias de execução.

Palavras-chave: Sepse. Unidades de terapia intensiva. Pressão venosa central.

\section{ABSTRACT}

Objective: To evaluate medical records of the patients with sepsis in the intensive care unit (ICU) of a tertiary hospital, measuring the degree of adherence to the sepsis protocol of brazilian Ministry of Health. Method: This was a cross-sectional and descriptive study that measured the frequency of adherence to intensive care within first 6 hours after diagnosis of sepsis. Results: We observed a predominance of male patients. The mean age of sample was $47 \pm 21$ years. Of all patients, 14 (28\%) were affected by sepsis, 21 $(42 \%)$ by severe sepsis and $15(30 \%)$ by septic shock. Of these patients, blood culture was collected in 28 (56\%), central venous pressure (CVP) in 47 (94\%), determination of the venous oxygen saturation index $\left(\mathrm{SVO}_{2}\right)$ in $5(10 \%)$ and measurement of plasma lactate in $42(84 \%)$. At the end, we found that in none of the patients all the strategies of protocol were performed. In addition, only in $4(8 \%)$ of them were implemented $80 \%$ of this protocol. Conclusion: In view of the lack of complete adhesion proposed in this protocol, it is necessary to increase the investment in the training of teams able to perform the strategies of execution.

Keywords: Sepsis. Intensive care units. Central venous pressure.

Autor correspondente: Rafael Hesley Araujo Costa, Rua Torres Câmara, 891, Aldeota, Fortaleza, Ceará. CEP: 60150-060. Telefone: +55 85 99927-5471. E-mail: rafael.hesley@hotmail.com

Conflito de interesses: Não há qualquer conflito de interesses por parte de qualquer um dos autores.

Recebido em: 08 Jun 2017; Revisado em: 01 Ago 2017; Aceito em: 08 Out 2017. 


\section{INTRODUÇÃO}

Atualmente, a sepse representa uma importante causa de morte no mundo, constituindo o maior problema de saúde em unidades de terapia intensiva (UTIs). Uma das justificativas para este fato tem sido o reconhecimento tardio dos sinais e sintomas precoces da sepse. ${ }^{1,2}$

A capacitação da equipe multiprofissional dos serviços médicos, através de treinamentos na execução de protocolos, visando o reconhecimento precoce da sepse, constitui uma estratégia essencial no diagnóstico e tratamento de pacientes sépticos. ${ }^{3}$ Assim como no trauma, infarto agudo do miocárdio e acidente vascular encefálico, o tratamento adequado nas horas iniciais da sepse influencia de forma significativa o desfecho clínico. ${ }^{4}$

As diretrizes internacionais para o tratamento de sepse e choque séptico de 2012 sugerem a ressuscitação imediata do paciente séptico, através da implementação de um pacote de 6 horas aplicado logo após o diagnóstico. Entre as medidas estabelecidas neste pacote incluem-se: hemoculturas, estudos de imagem no intuito de confirmar uma fonte potencial de infecção, medida de lactato nos 30 minutos iniciais e após as primeiras 6 horas, determinação do índice de saturação de oxigênio venoso misto $\left(\mathrm{SVO}_{2}\right)$ e infusão de volume, com a finalidade de atenuar a hipotensão. ${ }^{5}$

Com o objetivo de melhorar o tratamento de pacientes sépticos, o Ministério da Saúde tem estimulado os hospitais a aplicarem intervenções de forma precoce no tratamento da sepse, seguindo os pacotes de ressuscitação propostos no seu protocolo. ${ }^{1}$ Nesse sentido, ainda são escassos estudos que avaliem a dificuldade na adoção de um pacote de ressuscitação no Brasil, através de medidas precoces. Dessa forma, esse estudo propôs avaliar a adesão ao pacote de ressuscitação do paciente séptico nas primeiras 6 horas por parte da equipe de saúde.

\section{MÉTODO}

O presente trabalho constitui-se em estudo prospectivo, transversal e descritivo em hospital universitário público terciário (Hospital Geral César Cals de Oliveira) no período de julho de 2014 à fevereiro de 2015 (8 meses), através da avaliação dos prontuários de pacientes internados em UTIs. Incluíram-se indivíduos com dois ou mais dos seguintes critérios clínicos da síndrome da resposta inflamatória sistêmica: I) Temperatura corporal $>38^{\circ} \mathrm{C}$ ou $<36^{\circ} \mathrm{C}$; II) Frequência cardíaca $>90 \mathrm{bpm}$; III) Frequência respiratória $>20$ irpm ou pressão parcial de $\mathrm{CO}_{2}<32 \mathrm{mmHg}$; IV) Leucócitos $>12.000$ células $/ \mathrm{mm}^{3}$ ou $<4.000$ células $/ \mathrm{mm}^{3}$, ou ainda presença de $>10 \%$ de células jovens (bastões), na presença de foco infeccioso documentado ou fortemente suspeito. ${ }^{5}$ Pacientes com sepse grave apresentaram os critérios de sepse com um ou mais sinais de disfunção orgânica: a pressão arterial sistólica $<90 \mathrm{mmHg}$ ou pressão arterial média $(\mathrm{PAM})<65 \mathrm{mmHg}$, creatinina $>2 \mathrm{mg} / \mathrm{dL}$, contagem de plaquetas $<100.000 \mathrm{~mm}^{3}$, frequência respiratória $>40$ irpm, ou icterícia (bilirrubina $>2 \mathrm{mg} / \mathrm{dL}$ ), ou comprometimento da troca pulmonar (pressão parcial de oxigênio arterial/fração inspirada de oxigênio $<250 \mathrm{mmHg}$ ), PAM abaixo de 65 $\mathrm{mmHg}$, injúria renal aguda ( 1,5 vezes o aumento da creatinina basal ou diurese $<0,5 \mathrm{ml} / \mathrm{kg} / \mathrm{h}$ durante duas horas), ou hiperlactatemia. ${ }^{5}$ Já os pacientes sépticos refratários a infusão de cristalóides (persistiram com PAM $<65 \mathrm{mmHg}$ ), e com necessidade de agentes vasopressores foram classificados como choque séptico. ${ }^{5}$ Foram excluídos indivíduos com idade inferior a 14 anos. Foram relacionadas variáveis gerais de sexo, idade e sítio primário acometido. Nestes pacientes, foi determinada a frequência de adesão às medidas de cuidados intensivos nas primeiras 6 horas após diagnóstico de sepse, baseada no protocolo de sepse do Ministério da Saúde. ${ }^{6}$ As medidas consistiram em: checagem do lactato nos 30 minutos iniciais e após 6 horas, reposição volêmica baseada em metas (volume infundido $>500 \mathrm{ml}$ nos pacientes com lactato superior a 2,2), realização de acesso venoso central com a mensuração da saturação venosa central $\left(\mathrm{SVO}_{2}\right)$, coleta de hemoculturas e a mensuração da pressão venosa central (PVC). ${ }^{6}$ A cada um destes itens foi atribuído o percentual de $20 \%$ da meta desejada. Ao final, os dados do estudo foram avaliados usando o programa SPSS 13.0 for Windows, utilizando-se análises univariadas descritivas (frequência, média e desvio padrão). A pesquisa foi aprovada pelo Comitê de Ética do Hospital Geral César Cals de Oliveira e os aspectos éticos foram assegurados pelas Normas e Diretrizes Regulamentadoras da Pesquisa Envolvendo Seres Humanos - Res.466/12CNS/MS, sob o número 037897/2014.

\section{RESULTADOS}

Constatou-se que a maior parte da amostra era do sexo masculino, com 28 homens (56\%). A média de idade da amostra foi de $47 \pm 21$ anos. Do total, 14 (28\%) pacientes apresentaram sepse, 21 (42\%) sepse grave e 15 (30\%) choque séptico. O sítio infeccioso mais prevalente foi o pulmão, com $20(35 \%)$ casos, seguido de partes moles, com 12 (21\%) casos, trato gastrointestinal, com $10(17 \%)$ casos, sistema nervoso central, com $5(9 \%)$ casos e trato geniturinário, com 2 (4\%) casos. Apenas $1(2 \%)$ paciente não teve sítio de infecção determinado.

Com relação à adequação das medidas e o tempo em que foram realizadas após o diagnóstico de sepse, sepse grave ou choque séptico, constatou-se que somente 28 (56\%) pacientes realizaram hemocultura, das quais somente 22 (44\%) foram efetuadas nas primeiras 6 horas. Já em relação à PVC, 47 (94\%) realizaram o procedimento dentro das primeiras 6 horas. Constatou-se que $42(84 \%)$ dos pacientes tiveram o lactato plasmático dosado na primeira meia hora após o diagnóstico, e $24(48 \%)$ foram reavaliados após 6 horas, sendo possível avaliar a redução do lactato. Foi observado que em 13 (26\%) dos pacientes o lactato baixou em $50 \%$ ou normalizou com as medidas introduzidas nas 6 horas iniciais. Quanto a $\mathrm{SVO}_{2}$, esta só foi mensurada em $5(10 \%)$ dos pacientes nas primeiras 6 horas, embora todos tivessem acesso venoso central. 
O gráfico 1 mostra as diferentes faixas de infusão de volume, com 25 (50\%) dos pacientes recebendo menos de $500 \mathrm{~mL}$ de volume nas primeiras 6 horas.

Em nenhum dos pacientes foram realizadas todas as medidas propostas no protocolo de sepse. O gráfico 2 mostra os valores percentuais dos pacientes que foram submetidos a diferentes níveis de adesão ao protocolo.

Gráfico 1. Quantidade de volume infundido no pacote de 6 horas nos pacientes diagnosticados com sepse, sepse grave ou choque séptico.

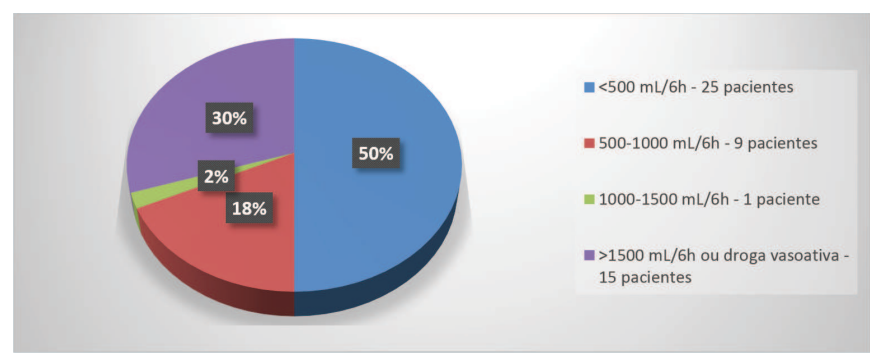

Gráfico 2. Percentual de parâmetros estabelecidos como meta no protocolo de ressuscitação de pacientes sépticos nas primeiras 6 horas após o diagnóstico de sepse.

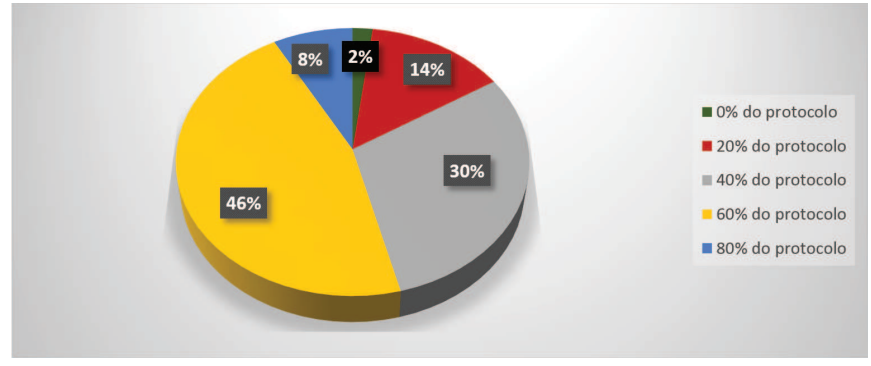

\section{DISCUSSÃO}

Os protocolos para o tratamento imediato de pacientes acometidos por sepse apresentam ampla aceitação nos Estados Unidos e na Europa. ${ }^{7}$ A administração agressiva de fluidos, o suporte hemodinâmico e as abordagens guiadas pela avaliação do lactato nas primeiras 6 horas após a admissão contribuem para melhores desfechos clínicos em pacientes com sepse. ${ }^{8}$

A ressuscitação inicial visa atingir as metas estabelecidas pelas "Diretrizes da campanha de sobrevivência à sepse para tratamento de sepse grave e choque séptico", nas quais o presente estudo se baseou. A adesão a essas diretrizes foi associada a uma redução absoluta de $15,9 \%$ na taxa de mortalidade após período de 28 dias, verificada em um estudo multicêntrico com 314 pacientes com sepse grave. ${ }^{9}$

No presente estudo, houve uma maior incidência de sepse no sexo masculino, totalizando 56\%. Este resultado está de acordo com o verificado em hospitais públicos brasileiros, onde nos últimos dez anos observou-se que, do total de pacientes acometidos por sepse, 56,5\% eram homens. ${ }^{10}$ Alguns estudos defendem que diferenças hormonais entre os sexos e níveis mais elevados de mediadores anti-inflamatórios influenciam consideravelmente o desfecho clínico. O estrógeno parece apresentar efeito protetor, através da modulação na resposta imune ao agente infeccioso. ${ }^{11}$ Paralelamente, alguns modelos experimentais de sepse têm sugerido papel relevante desse hormônio na redução do estresse oxidativo induzido por sepse, outro efeito que explicaria menor incidência e melhor prognóstico em pacientes do sexo feminino, observados comumente na literatura. ${ }^{12}$

No presente trabalho, observou-se que o sítio pulmonar constituiu o principal local de origem da sepse e de suas complicações, conforme observado em 35,1\% dos pacientes. Este resultado corrobora o encontrado em um estudo epidemiológico de sepse em UTIs brasileiras. ${ }^{13} \mathrm{~A}$ alta prevalência do sítio respiratório como fonte de infecção para sepse é compatível com a proporção de pacientes admitidos com doenças pulmonares, sobretudo com infecções respiratórias, além do número crescente de pacientes submetidos a ventilação mecânica em UTIs. ${ }^{14}$ Diferentemente do sítio acometido, a média de idade aqui encontrada $(47,7$ anos) foi bem inferior à de 61,7 e 64,4 anos encontrada em outros estudos epidemiológicos. ${ }^{15,16}$ Uma das razões para a discrepância na média de idade pode estar associada ao perfil do hospital em que foi realizado o estudo, onde há apenas emergência obstétrica e os internamentos clínicos e cirúrgicos são, em sua maioria, eletivos.

Através deste estudo, verificou-se que, em 44\% dos pacientes, a hemocultura não foi realizada em nenhum momento após o diagnóstico da sepse, o que representa um alto índice de não adesão desse procedimento. No intuito de obter o diagnóstico adequado, a coleta de sangue para hemocultura constitui uma estratégia na prática clínica, já que 50\% dos pacientes com sepse grave ou choque séptico apresentam hemoculturas positivas. ${ }^{17} \mathrm{O}$ caráter essencial da identificação imediata do agente etiológico está no fato de que determinar a terapia antimicrobiana adequada tem sido associado a um melhor prognóstico. ${ }^{18}$ Dessa forma, mostra-se essencial a realização da hemocultura prévia ao início do tratamento com antimicrobianos. Além disso, estes resultados são importantes no sentido de guiar futuras escolhas de esquemas terapêuticos empíricos para cada localização topográfica da infecção. ${ }^{4}$

Outra meta fisiológica avaliada no pacote de 6 horas foi a aferição da PVC. Neste estudo, foi possível determinar que, em $94 \%$ da amostra, foi realizado esse procedimento, o que caracteriza uma ótima adesão por parte da equipe médica, quando comparado a outros estudos. ${ }^{19,20} \mathrm{Um}$ estudo prévio

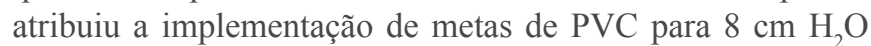
e da $\mathrm{SVO}_{2}$ acima de $70 \%$ durante as primeiras 6 horas de ressuscitação como um dos fatores determinantes para a redução na taxa de mortalidade de pacientes sépticos em UTIs brasileiras. $^{21}$

Apesar da ótima adesão à PVC, o mesmo não ocorreu com a determinação da $\mathrm{SVO}_{2}$, visto que em apenas $10 \%$ dos pacientes sépticos estudados foi realizada esta conduta. Assim, esta medida apresentou o menor índice de adesão em relação 
a todos os outros estabelecidos no pacote de 6 horas. Esse achado está de acordo com dados verificados pelo Instituto Latino Americano de Sepse, nos quais se observou que, entre 2004 e 2015 , em apenas $12,2 \%$ dos pacientes admitidos com sepse em hospitais públicos brasileiros a $\mathrm{SVO}_{2}$ foi realizada. ${ }^{10}$ Esses dados devem servir de alerta para a necessidade de imediato cumprimento da meta sobredita, que é fundamental no intuito de avaliar a perfusão e oxigenação tecidual.

Outras abordagens avaliadas no pacote de 6 horas foram a dosagem de lactato sanguíneo e o total de volume infundido nos pacientes. Apesar do lactato inicial ter sido medido na maior parte dos pacientes (42 dos 50), o lactato final foi medido em apenas 24 dos 50 pacientes, o que representa outra estratégia a ser melhorada, no intuito de garantir uma adequada ressuscitação volêmica dos pacientes com sepse. $^{22}$

Ademais, observou-se que $50 \%$ dos pacientes receberam fluido menor que $500 \mathrm{~mL}$, e $25 \%$ receberam volume superior a $1500 \mathrm{~mL}$ ou a administração de algum tipo de droga vasoativa. Durante a fase de ressuscitação do choque, a avaliação mais adequada da reposição volêmica se dá pela resposta cardiovascular à infusão hídrica. A avaliação desta auxilia na diferenciação entre a necessidade de intensificar a expansão volêmica e a indicação de inotrópicos para reversão da hipóxia tissular. ${ }^{23}$

\section{REFERÊNCIAS}

1. Silva E, Pedro MA, Sogayar AC, Mohovic T, Silva CL, Janiszewski $\mathrm{M}$, et al. Brazilian sepsis epidemiological study (bases study). Crit Care. 2004;8(4):251-60.

2. Conde KA, Silva CO, Ferreira E, Freitas FG, Castro I, Rea-Neto A, et al. Differences in sepsis treatment and outcomes between public and private hospitals in Brazil: a multicenter observational study. PLos One. 2013;8(6):e64790.

3. Micek ST, Roubinian N, Heuring T, Bode M, Williams J, Harrison $\mathrm{C}$, et al. Before-after study of a standardized hospital order set for the management of septic shock. Crit Care Med. 2006;34(11):2707-13.

4. Nguyen HB, Corbett SW, Steele R, Clark RT, Hayes SR, Edwards $\mathrm{J}$, et al. Implementation of a bundle of quality indicators for the early management of severe sepsis and septic shock is associated with decreased mortality. Crit Care Med. 2007;35(4): 1105-12.

5. Dellinger RP, Levy MM, Rhodes A, Annanne D, Gerlach H, Opal SM, et al. Campanha de sobrevivência à sepse: diretrizes internacionais para tratamento de sepse grave e choque séptico: 2012. Crit Care Med. 2013;41(2):580-637.

6. Brasil. Ministério da Saúde. Sociedade Beneficente Israelita Brasileira Hospital Albert Einstein. Instituto Latino Americano de Sepse. Programa de Apoio ao Desenvolvimento Institucional do Sistema Único de Saúde. Controlando a infecção, sobrevivendo a sepse. Brasília: Ministério da Saúde; 2012. 60 p.

7. Levy MM, Dellinger RP, Townsend SR, Linde-Zwirble WT, Marshall JC, Bion, J, et al. The surviving sepsis campaign: results of an international guideline-based performance improvement program targeting severe sepsis. Crit Care Med. 2010;38(2):367-74.
Finalmente, a taxa de adesão do protocolo proposto no pacote de 6 horas por parte da equipe médica no trabalho aqui estudado não atingiu os $100 \%$ em nenhum dos 50 pacientes. Outros estudos observaram baixa adesão do pacote completo de 6 horas, quando apenas em 4 de 66 pacientes foram realizados todos os procedimentos recomendados no plano de ressuscitação dentro das primeiras horas após detecção da sepse..$^{24}$

O processo de adesão de novos pacotes de ressuscitação por parte da equipe médica tem sido amplamente discutido. Falta de familiaridade, inércia da prática anterior, auto-eficácia, formato difícil e fatores ambientais como tempo insuficiente, recursos e barreiras institucionais constituem alguns dos exemplos que dificultam a execução prática de diretrizes adequadas para o tratamento da sepse. ${ }^{25}$

Portanto, em nenhum dos pacientes avaliados houve uma adesão integral às medidas propostas no protocolo de sepse do Ministério da Saúde. Ademais, observou-se uma baixa adesão geral à implementação de suas medidas, o que torna necessário um maior investimento na formação de equipes aptas a realizar suas estratégias de execução. Nesse sentido, sugere-se a criação de uma equipe de trabalho multidisciplinar, no intuito de comunicar, documentar e avaliar diariamente a execução do pacote de 6 horas. Além disso, a implementação de programas constantes de treinamento e de atualização da equipe mostra-se fundamental.

8. Cohen J, Vicent JL, Adhikari NK, Machado FR, Angus DC, Calantra T, et al. Sepsis: a roadmap for future research. Lancet Infect Dis. 2015:15(5):581-614.

9. Jing Y .The effect of early goal-directed therapy on treatment of critical patients with severe sepsis/septic shock: a multi-center, prospective, randomized, controlled study. Chin Crit Care Med. 2010;6:331-4.

10. Rhodes A, Evans LE, Alhazzani W, Levy MM, Antonelli M, Ferrer R, et al. Surviving sepsis campaign: International Guidelines for Management of Sepsis and Septic Shock: 2016. Crit Care Med. 2017;45(3):1-67.

11. Couto DO, Peixoto AA Júnior, Farias JL, Sales DB, Lima JP, Rodrigues RS, et al. Associação entre sexo e mortalidade em pacientes com sepse: os hormônios sexuais influenciam o desfecho? Rev Bras Ter Intensiva. 2003;23(3):297-303.

12. Sener G, Arbak S, Kurtaran P, Gedik G, Yegen, BC. Estrogen protects the liver and intestine against sepsis-induced injury in rats. $\mathrm{J}$ Surg Res. 2005;128(1):70-8.

13. Sales JA Júnior, David CM, Hatum R, Souza PC, Japiassu A, Pinheiro CT, et al. Sepse Brasil; estudo epidemiológico de sepse em Unidades de Terapia Intensiva brasileiras. Rev Bras Ter Intensiva. 2006;18(1):9-17.

14. Feijó CA, Leite JR, Martins AC, Furtado AH Júnior, Cruz LL, Meneses FA. Gravidade dos pacientes admitidos à Unidade de Terapia Intensiva de um Hospital Universitário Brasileiro. Rev Bras Ter Intensiva. 2006;18(1):18-21. 
15. Sales JA Júnior, David CM, Hatum R, Souza PC, Japiassú A, Pinheiro CT, et al. Sepse Brasil: estudo epidemiológico da sepse em Unidades de Terapia Intensiva brasileiras. Rev Bras Ter Intensiva. 2006;18(1):9-17.

16. Taniguchi LU, Bierrenbach AL, Toscano CM, Schettino GP, Azevedo LC. Sepsis-related deaths in Brazil: an analysis of the national mortality registry from 2002 to 2010. Crit Care Med. 2014;18(6):608.

17. Diament D, Salomão R, Rigatto O, Gomes B, Silva E, Carvalho NB, et al. Guidelines for the treatment of severe sepsis and septic shock: management of the infectious agent - diagnosis. Rev Bras Ter Intensiva. 2011;23(2):134-44.

18. Harbhart S, Garbino S, Pugin J, Romand JA, Lew D, Pittet D. Inappropriate initial antimicrobial therapy and its effecton survival in a clinical trial of immunomodulation therapy for severe sepsis. Am J Med. 2003;115(7):529-35.

19. Nguyen HB, Kuan WS, Batech M, Shrikhande P, Mahadevan M, $\mathrm{Li} \mathrm{CH}$, et al. Outcome effectiveness of the severe sepsis resuscitation bundle with addition of lactate clearence as a bundle item: multinational evaluation. Crit Care. 2011;14(5):R229.
20. Girardis M, Rinaldi L, Donno L, Marietta M, Codeluppi M, Marchegiano P, et al. Effects on management and outcome of severe sepsis and septic shock patients admitted to the intensive care unit after implementation of a sepsis program: a pilot study. Crit Care. 2009;13(5):R143.

21. Seymour CW, Rosengart MR. Septic shock: advances in diagnosis and treatment. Jama. 2015;314(7):708-17.

22. Corrêa TD, Rocha LL, Pessoa CMS, Silva E, Assunção MS. Fluid therapy for septic shock resuscitation: which fluid should be used? Einstein. 2015;13(3):462-8.

23. Andrews B, Muchemwa L, Kelly P, Lakhi S, Heimburger DC, Bernard GR. Simplified severe sepsis protocol: a randomized controlled trial of modified early goal-directed therapy in Zambia. Crit Care Med. 2014;42(11):2315-24.

24. Pereira AJ, Fernandes CJ Junior, Sousa AG, Akamine N, Santos GP, Cypriano AS, et al. Melhoria de desempenho e desfechos (mortalidade) após implementação de um protocolo institucional de atendimento a pacientes sépticos. Einstein. 2008;6(4):395-401.

25. Cinel I, Dellinger RP. Guidelines for severe infections: are they useful? Curr Opin Crit Care. 2006;12(5):483-88.

\section{Como citar:}

Costa RH, Costa PH, Macedo AL, Pessoa RM, Braga IL. A baixa adesão do protocolo de sepse na terapia intensiva. Rev Med UFC. 2018 jul-set;58(3):20-24. 